\title{
Postures lyriques. L'ajustement voix-rôle dans le travail interprétatif du chanteur soliste
}

In: Revue française de sociologie. 2000, 41-2. pp. 277-305.

Citer ce document / Cite this document :

Gonzalez Martinez Esther. Postures lyriques. L'ajustement voix-rôle dans le travail interprétatif du chanteur soliste. In: Revue française de sociologie. 2000, 41-2. pp. 277-305.

http://www.persee.fr/web/revues/home/prescript/article/rfsoc_0035-2969_2000_num_41_2_5262 


\section{Résumé}

Une définition interactionniste du travail comme ajustement entre des compétences et des rôles fonde notre étude de l'interprétation lyrique. L'analyse de notre matériel empirique - entretiens avec des chanteurs solistes et sources publiées - met en évidence un vaste processus de détermination réciproque au cours duquel il y a construction d'une voix en fonction d'un emploi donné et façonnement d'un rôle lyrique selon les compétences de l'artiste. Ce processus d'ajustement se concrétise dans l'adoption par le chanteur, au cours de la « production », d'une posture lyrique. L'interprétation lyrique prend forme au cours des interactions que le chanteur établit avec les autres membres de la « production »; elle varie selon les caractéristiques et les interrelations des trois dimensions - vocale, musicale et dramatique - de la posture, ainsi que selon la résolution donnée aux tensions qui la traversent.

\section{Resumen}

Esther González Martínez : Posturas líricas. La adaptación voz-personaje en el trabajo interpretativo del cantante lírico.

Nuestro estudio de la interpretación lírica se basa sobre una definición interaccionista del trabajo como adaptación entre las competencias y los roles. El análisis del material empírico - entrevistas con los cantantes líricos y de las fuentes publicadas - pone en evidencia un amplio proceso de determinación recíproca en el cual hay construcción de una voz en función de un empleo determinado y de la elaboración de un personaje lírico de acuerdo a las competencias del artista. Este proceso de adaptación résulta de la adopción por el cantante de una postura lírica en el curso de la « producción ». La interpretación lírica toma forma durante las interacciones que el cantante establece con los otros miembros de la « producción ». Puede variar de acuerdo a las características y a las interelaciones de tres dimensiones - vocal, musical y dramática -, también de la postura, así como de la resolución dada a las tensiones que la atraviezan. determinación recíproca en el cual hay construcción de una voz en función de un empleo determinado y de la elaboración de un personaje lírico de acuerdo a las competencias del artista. Este proceso de adaptación résulta de la adopción por el cantante de una postura lírica en el curso de la " producción ». La interpretación lírica toma forma durante las interacciones que el cantante establece con los otros miembros de la « producción ». Puede variar de acuerdo a las características y a las interelaciones de tres dimensiones - vocal, musical y dramática -, también de la postura, así como de la resolución dada a las tensiones que la atraviezan.

\section{Zusammenfassung}

Esther Gonzáles Martínez : Lyrische Haltungen. Die Anpassung von Stimme und Rolle in der Interpretationsarbeit der Gesangssolisten.

Unsere Untersuchung zur lyrischen Interpretation stützt sich auf eine interaktionistische Definition der Arbeit als Anpassung zwischen Kompetenzen und Rollen. Die Analyse unseres empirischen Materials : Unterredungen mit Gesangssolisten und Veröffentlichungen - macht einen weiten wechselseitigen Determinierungsprozess sichtbar, bei dem eine Stimme aufgebaut wird abhängig von der gegebenen Rolle, sowie die Herausbildung einer lyrischen Rolle entsprechend den Kompetenzen des Künstlers. Dieser Anpassungsprozess entsteht aus der Einnahme durch den Sänger, im Verlauf seiner " Produktion » einer lyrischen Haltung. Die lyrische Interpretation bildet sich heraus im Verlauf der Interaktionen, die der Sänger mit den übrigen Mitgliedern der «Produktion » herstellt. Sie zeigt Abweichungen entsprechend den Eigenschaften und Zwischenbeziehungen der drei Dimensionen der Haltung - vokal, musikalisch und dramatisch - und auch entsprechend der Auflösung, die den sie durchlaufenden Spannungen gegeben wird.

Abstract

Esther González Martínez : Operatic postures. The adjustment of voice to role in the interpretation work of a solo singer.

An interactionist definition of work as the adjustment between competence and roles is the base of our 
study on operatic interpretation. The analysis of our empirical material - interviews with the solo singers and published sources - clearly shows a vast process of mutual determination during which a voice is constructed around a particular job and development of an operatic role according to the competence of the artist. This process of adjustment is the result of an operatic posture adopted by the singer during the " production ». The operatic interpretation takes on its different forms as a result of interactions built up between the singer and other members of the "production "; it varies according to the characteristics and interrelations of the three dimensions - vocal, musical and drama - of the posture, and in relation to the resolution given to the tensions which go through it. by the singer during the " production ». The operatic interpretation takes on its different forms as a result of interactions built up between the singer and other members of the " production »; it varies according to the characteristics and interrelations of the three dimensions - vocal, musical and drama - of the posture, and in relation to the resolution given to the tensions which go through it. 


\title{
Esther GONZÁLEZ MARTÍNEZ
}

\section{Postures lyriques}

\section{L'ajustement voix-rôle dans le travail interprétatif du chanteur soliste*}

\begin{abstract}
RÉSUMÉ
Une définition interactionniste du travail comme ajustement entre des compétences et des rôles fonde notre étude de l'interprétation lyrique. L'analyse de notre matériel empirique entretiens avec des chanteurs solistes et sources publiées - met en évidence un vaste processus de détermination réciproque au cours duquel il y a construction d'une voix en fonction d'un emploi donné et façonnement d'un rôle lyrique selon les compétences de l'artiste. Ce processus d'ajustement se concrétise dans l'adoption par le chanteur, au cours de la «production », d'une posture lyrique. L'interprétation lyrique prend forme au cours des interactions que le chanteur établit avec les autres membres de la «production »; elle varie selon les caractéristiques et les interrelations des trois dimensions - vocale, musicale et dramatique - de la posture, ainsi que selon la résolution donnée aux tensions qui la traversent.
\end{abstract}

L'activité lyrique a essentiellement été abordée selon des approches musicologiques, historiques ou économiques. Les traits formels des voix et leurs emplois (Celletti, 1987 ; Leibowitz, 1997 ; Moindrot, 1993a), les conditions de travail et la figure de l'artiste lyrique à travers les temps (Bianconi et Pestelli, 1992 ; Lacombe, 1997 ; Roselli, 1984, 1997), le management des maisons d'opéra ou le fonctionnement des marchés lyriques (Bovier-Lapierre, 1988 ; Towse, 1993, 1997 ; Towse et Khakee, 1992) comptent au nombre des aspects ainsi étudiés (1). Les caractéristiques du métier de chanteur lyrique - formation

* Le professeur Alfred Willener et Roberta Shapiro ont guidé nos premières investigations ; nous avons présenté les résultats de notre recherche à l'université de Genève, à l'invitation du professeur André Ducret ; les professeurs JeanYves Pidoux et Louis Quéré, Olivier Giraud, Laurence Kaufmann, Muriel Surdez et Marilène Vuille ont lu, commenté et corrigé les différentes versions de ce texte ; le professeur André Petitat nous a libérée de nos obligations académiques pour nous permettre de cultiver nos «passions lyriques » ; les remarques des membres du comité de rédaction de la Revue française de sociologie, particulièrement d'Emmanuèle Reynaud et de Pierre-Michel Menger, nous ont aidée à orienter et à approfondir de façon substantielle nos analyses ; qu'ils soient ici tous très chaleureusement remerciés.

(1) La description et l'analyse du travail de chanteur a par ailleurs été l'apanage des artistes, agents, professeurs de chant ou journalistes spécialisés, participants directs au monde lyrique dont les écrits portent généralement sur les aspects les plus techniques du travail artistique, ou 
très spécifique, haut degré de professionnalisation, postes et lieux de travail en nombre relativement limité, «typicité» des trajectoires, etc. - le distinguent d'autres activités artistiques à l'organisation plus difficilement saisissable, mais ne lui ont valu jusqu'ici qu'un faible intérêt de la part des sociologues. Malgré les travaux de Kamerman et Martorella (1983), Martorella (1982) ou Richardson (1980), l'activité de chanteur d'opéra fait encore figure, pour notre discipline, de terra incognita (2).

Dans cet article nous chercherons à pénétrer, dans une perspective sociologique, au cœur même de la pratique des solistes, en abordant le thème de l'interprétation lyrique que nous situerons dans son milieu d'occurrence privilégié : le spectacle d'opéra. Nous proposerons un cadre d'analyse susceptible d'appréhender les constituantes essentielles du travail interprétatif tout en l'inscrivant dans un processus de construction de l'ajustement voix-répertoire qui dépasse les limites de la représentation scénique. Nos conclusions sont issues principalement de l'analyse d'un corpus constitué d'entretiens avec des chanteurs - réalisés dans le cadre d'une recherche collective sur des interprètes musiciens - ainsi que de sources publiées telles qu'interviews, biographies et mémoires (voir Annexe I).

Pour l'artiste performant «produire» équivaut à se produire. Devant son public, le chanteur est tenu d'incarner un rôle du répertoire. Sa prestation, expression directe du corps, participe à l'élaboration d'une œuvre collective, la représentation d'opéra, dont elle tire son sens et par rapport à laquelle les différentes contributions individuelles - chant, interprétation instrumentale, mise en scène - prennent forme. Ce constat de base permet de mettre en évidence trois médiateurs (3) lyriques: les compétences, les rôles et les «productions » (4). Ils servent aux chanteurs à élaborer leurs interprétations et à assurer la communication avec le public. Notre recherche est organisée autour d'un principe central : étudier l'interprétation comme une concrétisation scénique d'un processus d'appariement et d'ajustement entre des compétences et des rôles, au cours duquel ces deux entités se déterminent réciproquement. Les interactions du chanteur avec son environnement assurent l'ajustement et sont à la base des opérations de formation, modulation et manifestation des interprétations.

(suite de la note 1)

sur telle diva ou tel événement particulier. Dans cette littérature se distinguent quelques ouvrages plus généraux, voir Christiansen (1984); The future of opera (1986) ; M. Harries et S. Harries (1986).

(2) Des sociologues se sont intéressés à d'autres aspects de l'opéra: Adorno (1994) défend l'hypothèse selon laquelle la forme opera serait en crise parce qu' incapable de communiquer avec son public ; Salazar (1980) présente l'évolution de l'opéra comme une suite articulée de systèmes de pensées conjoints à la société ; Urfalino (1990) retrace la naissance agitée de l'Opéra-Bastille.

(3) Au sens de Hennion (1993).

(4) Dans le monde lyrique, l'expression «production» désigne la mise en scène d'une cuvre : il y a de nouvelles productions et des reprises. Par «production» (entre guillemets) nous entendons un cours d'action collectif et concerté au sein duquel s'effectuent toutes les opérations nécessaires à la représentation d'un opéra. 
$\mathrm{Au}$ croisement de l'approche interactionniste et des théories de l'action pratique, nous avons formulé un cadre d'analyse susceptible d'éclairer la facette interprétative du travail de soliste, dont la présentation fera l'objet de la première partie de cet article (5). Nous porterons ensuite notre attention sur la nature, composite et extrêmement sujette à changement, de deux entités - la voix et les rôles lyriques - en mettant en évidence le rapport dynamique de détermination réciproque qui les unit. Le processus d'ajustement se traduit par la construction d'une posture : réponse du chanteur à l'œuvre et à la «production », mise en voix et mise en corps du texte musical et dramatique. Nous exposerons d'abord les dimensions de ces postures lyriques, qui signifient les interactions du chanteur avec les membres de la «production», et nous terminerons par une présentation des tensions qui les habitent et les façonnent. Tout au long de notre travail nous avons veillé à rendre au processus d'ajustement son caractère pratique, dynamique et «fait en interaction».

\section{Éléments pour un modèle pratique de l'interprétation lyrique}

«...[L]'accomplissement du travail à l'intérieur d'une organisation est un acte collectif coordonné. » (Corbin et Strauss, 1993, p. 74) (6). La production d'un spectacle lyrique peut être assimilée à une entreprise collective visant à représenter sur scène une pièce du répertoire, et dont la réalisation oriente, de manière plus ou moins prégnante, le sens des différentes contributions individuelles. Dans ce contexte, des espaces d'action, que l'on pourrait appeler des positions, sont ouverts, et leurs occupants chargés de la concrétisation d'une partie du projet : direction d'orchestre, chant, éclairages, régie, etc. À chaque position, et à ses objectifs particuliers, correspondent des modes d'action et de comportement spécifiques : les rôles. Des auteurs tels que Goffman, Strauss ou Hughes ont considéré les activités professionnelles comme des occasions de rencontre entre les identités des acteurs et les rôles qui leur sont proposés sur les lieux de travail. Cette rencontre déclenche un processus au cours duquel les acteurs procèdent à l'expression, à la reconnaissance, à la construction et à la modification des identités et des rôles en vue de leur ajustement réciproque. Des différentes définitions de l'identité formulées par ces auteurs, nous retiendrons que celle-ci se traduit dans des aptitudes et des dispositions à agir particulières (Goffman, 1973, 1975), par exemple les aptitudes et dispositions dramatiques, vocales et musicales du chanteur. Pour ce qui est des rôles, Turner refusait leur assimilation à des instructions dont le respect assurerait l'accomplissement de

(5) Dans un de ses derniers articles, Strauss préconisait des études encore plus détaillées que celles effectuées auparavant sur la réalisation concrète, au sein et grâce à des interactions, des activités professionnelles (Corbin et Strauss, 1993). On retrouve dans les théories de l'action pratique (Pharo et Quéré, 1990 ; Quéré, 1993) des éléments utiles à la poursuite du programme interactionniste. Sur le possible renouvellement de la conceptualisation du travail à partir des théories de l'action, voir Cottereau (1994).

(6) Traduit par l'auteur. 
la tâche en question et avançait la notion de role taking/role making: un processus «...au cours duquel les rôles sont identifiés et prennent un contenu sur des axes changeants, à mesure que l'interaction se déroule » (1962, p. 27) (7). Les acteurs, dans un jeu d'influences croisées et en fonction de leurs intérêts, dispositions et possibilités d'action particulières, participent à une définition des rôles et de la façon de les exécuter.

Différents auteurs (Menger, 1989, 1991, 1994 ; Santos, 1976 ; Towse, 1993) ont traité de l'incertitude qui pèse sur l'exercice des métiers artistiques en général, et lyriques en particulier. Notre cadre d'analyse entre en résonance avec ces travaux pour situer l'incertitude au niveau même du façonnage du produit artistique (8). La construction de l'interprétation, ce processus où se modulent rôles et identités, est considérée comme un «cours d'action » dont les acteurs ne peuvent pas réduire l'indétermination avant d'y participer. La notion d'interprétation, appliquée au théâtre, à la musique ou à la danse, se distingue de celle d'exécution et exprime le fait de donner corps concrètement à ce qui n'existe qu'à l'état de possible. Tant qu'une voix et un rôle ne se rencontrent pas, leur nature n'est pas pleinement déterminée. La voix devient chant grâce à la partition, l'écriture musicale peut se transformer en musique grâce à l'artiste. C'est bien d'une «production » qu'émerge une concrétisation pratique du rapport voix-rôle. Le chant, l'interprétation surgissent d'une rencontre où ces deux entités se déterminent réciproquement en fonction de leurs caractéristiques ainsi que des circonstances concrètes dans lesquelles cette rencontre a lieu (9). Par ailleurs, le partenariat d'acteurs, aux orientations et motivations souvent contradictoires, produit des innovations, entraîne des imprévus, chacune de leurs actions déterminant de nouveaux possibles (10). Le chanteur s'engage dans un cours d'action qui se révèle, se précise et se développe à mesure que le temps passe, et où il est question notamment, pour ce qui concerne l'artiste, de la détermination de deux entités liées : les caractéristiques du rôle et les compétences nécessaires à son interprétation.

L'ajustement, bon ou mauvais, s'effectue à chaque «production », son «avènement» est le propre d'une action coordonnée. Néanmoins l'artiste a d'excellentes raisons, ne serait-ce que du seul point de vue de l'interprétation, pour souhaiter un ajustement réussi. C'est de lui que dépend cette chose difficile à saisir, la «qualité » de l'interprétation; et plus concrètement, il y va de la

(7) Traduit par l'auteur.

(8) Menger (1994, 1997b) réfléchit à l'emploi en tant qu' «....appariement entre les caractéristiques du postulant et celles du travail à accomplir » (1997b, p. 67). Son approche reprend les théories économiques du «job matching»; nos références proviennent davantage du courant interactionniste.

(9) Dans cette perspective, l'interprétation est l'accomplissement et la résultante d'un cours d'action. Concrétisation, actualisation, ces ter- mes renvoient à l'herméneutique littéraire et plus particulièrement à l'école de Constance (Jauss, 1978). Pour une analyse sémiotique de l'interprétation et de la réception des arts de la performance, voir les travaux de Pavis (1983) sur le théâtre, et le livre de Moindrot (1993b) sur l'opéra.

(10) Nos développements sur l'indétermination des cours d'action et la réduction de l'incertitude s'inspirent de Quéré et Relieu (1997), et Relieu et Quéré (1998). 
préservation de sa voix (11). En effet, déterminer les «limites vocales» et maîtriser le risque de défaillance sont des principes pratiques conditionnant de façon prépondérante la correspondance voix-répertoire ainsi que les circonstances concrètes de représentation.

Goffman utilise les concepts de footing, posture et stance pour rendre compte de la façon dont un rôle est concrètement accompli dans une situation donnée (1968, 1987). Corbin et Strauss (1993) donnent une signification similaire au terme stance lorsqu'ils analysent la réalisation concrète du travail à travers des interactions. Nous dirons que le processus d'ajustement se traduit, pour ce qui est du chanteur, par la construction et l'adoption d'une posture. Ce dernier concept fait référence à la façon qu'a l'artiste d'habiter le rôle, de se l'approprier, de «couler» son identité dans l'emploi qu'il occupe. La posture est la réponse du chanteur à l'œuvre et à la «production »; entité mouvante, elle reflète - de façon souvent indirecte - les circonstances de l'appariement. C'est par sa posture que l'interprète va énoncer un point de vue particulier - à la fois personnel et collectif, contextualisé et irréductible au contexte - sur l'œuvre qu'il interprète.

Les interactions (dont les interactions face-à-face ne sont qu'un spécimen particulier) fondent les opérations de modulation et de manifestation des interprétations. Ceci posé, comment rendre compte de cette construction, en interaction, des interprétations lyriques ? Les échanges, les pratiques, les négociations ou les actions stratégiques sont «la matière» à partir de laquelle se constituent les processus qui intéressent véritablement le sociologue ; en conséquence leur description exhaustive ne paraît pas la démarche optimale pour rendre compte de notre objet: la nature intime du travail lyrique. Réfléchir en termes de processus exige de se concentrer sur l'équivalent lyrique de la circulation (pour utiliser une métaphore fort prosaïque mais chère à Goffman) : le cours d'action et son résultat, l'ajustement voix-rôle dont émergent les postures lyriques. C'est ainsi que nous avons voulu «transcender» non seulement les pratiques, mais aussi les «conducteurs», les «véhicules» et leurs «moteurs», les «règles de conduite» et autres «forces institutionnelles», pour mieux rendre compte, de l'intérieur, de la dynamique propre à l'activité interprétative, en signalant ses composantes et ses virtualités (12).

(11) Nous avons traité de cette question dans González Martínez (1998). De nombreux écrits témoignent de la préoccupation du monde lyrique pour les défaillances vocales : The future of opera (1986), Saint-Pulgent (1991).

(12) «Goffman ne fonde pas sa réflexion, comme on le fait habituellement, sur des agents individuels et des structures sociales auxquelles les agents doivent se conformer ou résister. Il commence plutôt par ces arrangements, engagements et ententes qui permettent aux agents et aux structures sociales d'avoir d'abord une existence sociale. [...] Goffman présente un portrait des contraintes de l'interaction qui sont internes aux situations d'interaction elles-mêmes. » (Rawls, 1987, pp. 139-141) [traduit par l'auteur]. Les théories de l'action reprennent l'idée de Goffman - obscure, critiquée, mais fort inspiratrice - de l'ordre de l'interaction comme domaine d'étude autonome et analytiquement viable. Un ordre de l'interaction dont l'organisation n'est pas tant à rapporter à des entités externes - forces institutionnelles ou choix individuels - qu'à décrire soigneusement depuis l'intérieur. 


\section{De l'indétermination à la construction d'une posture}

Les années de formation et les différentes expériences professionnelles participent à la préparation de chacune des interprétations du chanteur. Le processus d'ajustement voix-rôle ne débute en conséquence pas avec la «production ». Il s'agit d'une entreprise plus vaste, dont nous spécifions ici la nature et quelques-uns de ses aspects (il ne s'agit pas d'étapes) en en faisant ressortir le caractère «fait en interaction». En guise de préalable à l'étude des postures, il s'agit de combattre l'illusion de la naturalité des compétences et de la fixité de l'œuvre pour montrer comment ces deux entités se déterminent réciproquement. Notre intention n'est pas d'étudier les trajectoires ou les carrières, mais de définir le processus général - sa nature et sa dynamique - qui constitue et sous-tend toute configuration du travail lyrique (13).

\section{La voix, de la nature à l'emploi (14)}

«Le chant est une conquête de tous les instants. C'est au travail que je dois le développement, et même l'éclosion, de ma voix.» (15). Les digressions sur l'inné, le don et la révélation - l'idée d'une «voix naturelle »-propres à une conception romantique et charismatique de l'artiste sont contredites par grand nombre de déclarations des chanteurs, et surtout par leurs pratiques, qui témoignent d'une véritable entreprise de construction et d'intériorisation des compétences.

Le terme embodiment (Mead, 1963) convient pour décrire le travail d'inscription de la dynamique musicale dans le corps de l'artiste. Les années d'apprentissage et le temps consacré à la préparation d'un rôle visent à former un corps, un instrument qui réponde aux injonctions données par la partition - voire les devance : «...je n'ai qu'à penser à un rôle et ma gorge prend inconsciemment les positions correctes sans que j'aie besoin de chanter vraiment. » (16). L'embodiment se traduit par la mise en place de mécanismes précis de production du

(13) Notre objectif, bâtir un cadre dans lequel appréhender globalement l'interprétation, s'accorde aux caractéristiques de notre corpus très hétérogène -, ce qui nous autorise à parler de « chanteurs solistes d'opéra » en termes généraux sans faire d'autres distinctions que ponctuelles. En revanche, étudier les carrières lyriques (trajectoires, étapes, sphères d'activité, univers professionnels, marchés du travail) qui présentent de nombreuses particularités en fonction du pays, du répertoire, du système de production, de l'époque, du degré de notoriété de l'artiste, etc., ou élucider les principes du recrutement, de l'ascen- sion professionnelle et de l'obtention de la reconnaissance lyrique demanderait un tout autre type de données et de recherche que celles présentées ici.

(14) Notre travail dissèque la nature composite de l'identité lyrique; dans cette partie, il s'agit principalement de la «voix » parce que c'est sur elle que pèse le plus lourdement l'illusion de la naturalité des compétences.

(15) Dolora Zajick, Opéra international (juillet-août 1995, p. 30).

(16) Hildegard Behrens dans Matheopoulos (1992, p. 50) [traduit par l'auteur]. 
son. L'acquisition et la mobilisation de cette «pensée incarnée », que les chanteurs associent à «avoir une bonne technique», sont un réquisit indispensable au couplage du corps et de la partition : «Si on est démuni de technique, on a beau savoir ce que c'est que l'amour, on peut le ressentir, on ne peut pas le traduire. Donc, il faut le moyen de le traduire et ça, c'est la règle de tout. » (Ténor, $\left.\mathrm{n}^{\mathrm{O}} 11\right)$.

Selon l'expression de l'un de nos interviewés, le professionnel a «...une vue pertinente sur ce qu'il veut faire avec sa voix » (Ténor, $n^{\circ} 11$ ). L'appropriation de la technique lyrique s'accompagne de la détermination et de la définition des caractéristiques vocales du chanteur. Comme l'enfant qui ne prend conscience des limites et des possibilités de son corps que par le contact avec les objets environnants, l'artiste doit se «frotter» aux partitions, à leurs exigences, ressentir la résistance que son corps leur oppose, pour saisir quelles sont les virtualités de son instrument et comment les développer et les concrétiser. La formation d'une voix consiste à distinguer et à souligner certains de ses traits et à déterminer avec précision l'emploi que l'on entend lui donner. Dans cette entreprise, les voix ayant précédé celle de l'artiste jouent un rôle capital. En guise d'exemple, voici comment Walter Legge, chef du département de musique classique d'EMI, décrit la «construction» de la voix de son épouse, Elisabeth Schwarzkopf : «Je décidai d'abord d'affiner, à l'aide d'enregistrements, l'idée qu'elle avait des possibilités vocales. Le timbre crémeux, épais au goût de porto millésimé et la noblesse de ligne de Rosa Ponselle; l'éclat slave de Nina Koshetz ; quelques extraits de la Carmen de Farrar, dont on allait retrouver plus tard quelques traces ; un seul mot de Melba : "Bada" [...] À partir de l'analyse de ce que nous trouvions de plus admirable dans ces différents modèles, nous faisions notre propre synthèse, la plus adaptable à ce que nous croyions pouvoir développer le mieux [dans] sa voix, pour le répertoire sur lequel nous allions nous concentrer.» (17).

La spécification progressive de la voix, qui fait ressortir ses traits typiques, correspond à une catégorisation. Le travail de façonnement mobilise et actualise un grand nombre de référents collectifs, telles les catégories vocales: une chanteuse qui se veut soprano dramatique tâchera de renforcer son médium et ses graves, d'augmenter le volume de sa voix et d'assombrir son timbre (18). Mais c'est notamment par rapport à des répertoires, des styles et des compositeurs que se fait la catégorisation de la voix : «Mefistofele, comme Zaccaria de Nabucco ou Philippe II, m'imposerait la recherche d'une couleur vocale et d'une approche psychologique radicalement différentes de celles exigées par les emplois rossiniens. Quant à la technique proprement dite, il me faudrait arrondir la voix, lui donner davantage de volume, en sacrifiant l'élasticité de l'émission propre au bel canto.»(19). Pour rendre compte de cette détermination, le

(17) Walter Legge dans Elisabeth Schwarzkopf (1983, p. 114).

(18) Voir González Martínez (1998) et «Classification des voix» (en Annexe I). Les textes de Chuilon (1994) et de Leibowitz (1997) contiennent des pages très éclairantes sur l'évolution de la classification vocale et des exigences des rôles.

(19) Michele Pertusi, Opéra international (septembre 1996, p. 8). 
chanteur nuance les catégories vocales; il se dira «contralto colorature rossinienne», «baryton Verdi », ou «soprano dramatique wagnérienne» (20). Le chanteur s'identifie à des catégories qui le décrivent sur plusieurs plans - vocal, dramatique, musical, professionnel. Du fait qu'elles sont organisées en réseau, en couples, en oppositions, en hiérarchies - voix baroque/voix d'opéra, soprano noble/soubrette, premier soprano/second soprano, etc. - ces catégories dessinent une multitude de liens entre le chanteur et son entourage. La catégorisation, qui oriente le processus d'ajustement, guide les attentes, les pratiques et les interactions entre les individus, et fait en définitive plus que rendre identifiable le chanteur : elle l'inscrit dans un ordre social.

Par l'intermédiaire de ces opérations, le chanteur se dote d'une sorte d'identité, définie principalement en termes de compétences, qui le caractérise en tant que professionnel et artiste, et le différencie de ses collègues. Rappelons-nous que le chanteur se trace une voie au sein d'un répertoire limité et connu, dans l'interprétation duquel il a été précédé par d'illustres collègues; de ce fait, la comparaison entre artistes est aisée. La prise de rôle est un des moments clés de l'individuation, un moment par lequel le chanteur suit les pas d'un prédécesseur, se distancie de la tradition ou affiche ses ambitions, bref, donne forme autant à son profil vocal qu'à son projet artistique et professionnel.

Une attribution de caractères de désidérabilité oriente, plus qu'elle ne le détermine, le processus de construction vocale. Elle s'exerce sur les rôles et les voix et permet d'opérer une sélection parmi le répertoire disponible de possibilités. En effet, pour de multiples raisons d'ordre musical, économique, affectif ou autre, un rôle acquiert une valeur «intrinsèque» susceptible d'attirer un chanteur. Il en est ainsi des parties qui constituent les rôles principaux d'opéras, souvent représentés dans les grands théâtres, qui forment «le vrai répertoire» ou des rôles qui multiplient les occasions de briller sur scène ou tout simplement de ceux qui sont sympathiques aux yeux du chanteur. Les types de voix n'échappent pas non plus à l'évaluation. Ainsi, les véritables ténors dramatiques étant rares et leurs rôles extrêmement difficiles, le jeune chanteur qui s'aventure dans ce répertoire peut être certain d'être généreusement récompensé (21). Du côté des cantatrices, il est notoire que c'est à la soprano qui parvient à accompagner ses aigus vertigineux d'un beau médium que reviendra la place de l'héroïne d'un grand nombre de pièces : «Mon Dieu, j'aurais tant voulu être une prima donna ! [...] Rien d'étonnant si je me suis donnée du mal pour en devenir une ! Avec mes divers rôles de soprano, Fidelio, la Maréchale du Chevalier à

(20) Un exemple cxtraordinaire de l'interrelation entre voix et répertoire est Joan Sutherland qui, à la fín des années cinquante, après avoir été mezzo, soprano dramatique et lyrique, «ressuscita » la voix de soprano colorature romantique et, dans le même mouvement, réintroduisit dans la programmation des grands théâtres les œuvres romantiques de Donizetti et de Bellini (Major, 1994).
(21) Nous pouvons déduire la composition du «vrai répertoire», comme le fait Martorella (1982), en partant des statistiques sur les opéras joués dans les grands théâtres. Saint-Pulgent (1991) donne de nombreuses informations sur la standardisation du répertoire. On peut trouver des renseignements sur les cotes des types vocaux dans Martorella (op. cit.). 
la rose, Kundry de Parsifal ou Ariane de l'opéra du même nom de Richard Strauss, j'y suis presque arrivée ! (Ludwig, 1996, pp. 117-118).

L'ajustement voix-rôle fait l'objet de constantes opérations de maintien et de reformulation. À moins que le chanteur poursuive la spécialisation à outrance, il craint de se limiter sur le plan vocal, musical ou théâtral, à un seul emploi, en raison du risque de se voir cataloguer dans un rôle et de mettre en péril des ressources vocales sollicitées par un seul et même registre. Il cherchera alors des rôles lui permettant de dévoiler de nouvelles facettes de sa personnalité artistique. En outre, de par son substrat physiologique, la voix est continuellement soumise au changement; elle se modifie avec l'âge, l'entraînement ou à la faveur d'une nouvelle technique. Ces changements demandent à l'artiste de veiller constamment, en empruntant de nouveaux rôles, à la correspondance entre sa voix et son répertoire. En définitive, la construction d'une voix se traduit par un réaménagement des classements précités - catégories vocales, répertoires - en fonction des possibilités vocales du chanteur, de son projet professionnel et des occasions précises d'emploi. Les chanteurs expriment ce travail pour se positionner par rapport à la constellation des rôles lyriques; ils mesurent la distance qui les sépare de certains rôles, tracent des raccourcis, définissent des points d'aboutissement, déclarent certaines parties hors de leur portée : «... et puis il y a des choses utopiques que je ne pourrai jamais chanter parce que c'est pas dans ma voix... Verdi ou Puccini... c'est pour une voix dramatique... lorsqu'on a une voix lyrique il faut attendre pour chanter ça... je rêverais de chanter... je ne sais pas Azucena par exemple... quelque chose d'énorme. » (Soprano, $n^{\circ} 2$ ).

L'ajustement est à faire et à refaire tout au long de la carrière d'un chanteur. Il commande la gestion temporelle des prises de rôle et des engagements ainsi que la gestion du «portefeuille d'activités » (22). «Mais je pense que dans une saison il faut alterner des rôles qui développent le grave, avec ceux qui développent l'aigu, ceux qui demandent un travail de souplesse, je ne sais pas : Mozart, ou faire une passion de Bach, et un rôle qui au contraire vous fait utiliser toutes vos possibilités à peine un cheveu en dessus. » (Soprano, $\mathrm{n}^{\circ} 5$ ). Lorsqu'il s'agit d'approcher un nouveau personnage et de parvenir à l'inscrire dans leur répertoire, les chanteurs dépensent des trésors d'ingéniosité tactique : jouer l'œuvre en version concert et ensuite à l'opéra, enregistrer d'abord en studio avant d'interpréter le rôle au théâtre, se familiariser avec les airs en les chantant en récital... (23). Tout au long de leur carrière, les artistes cherchent à alterner des engagements exigeants avec de plus légers, combinent l'activité d'opéra avec le

(22) Menger (1991, 1994) a repris la théorie économique du «choix de portefeuille » pour étudier comment l'artiste intermittent diversifie les risques économiques liés à sa pratique professionnelle et accumule simultanément du capital humain.

(23) Dans cet article, nous nous sommes limité à étudier l'interprétation au théâtre. Le studio d'enregistrement, le récital ou la radio sont des « milieux protégés » où le chanteur peut tester un nouveau rôle, se profiller comme un éventuel interprète d'un personnage donné, ou diversifier son répertoire en éludant les contraintes de la scène - ce qui expliquerait les différences notables qui existent entre les répertoires scéniques et ceux enregistrés. Un bon exemple est Elisabeth Schwarzkopf qui, pour la scène, réduit son répertoire pratiquement à six rôles (Fiordiligi, Donna Elvira, la Comtesse Almaviva, la Maréchale, la Comtesse de Capriccio et Alice de Falstaff), alors 
récital, les oratorios, les enregistrements ou l'enseignement, ménagent soigneusement dans leurs agendas du temps libre pour se reposer et organisent leurs saisons de façon à travailler les différents registres de la voix et à préparer progressivement leurs rôles; ils assurent ainsi, en définitive, l'ajustement de leur voix aux rôles qu'ils interprètent.

\section{Un entrelacs de pratiques et d'acteurs}

La voix d'un chanteur, loin d'être donnée naturellement, résulte d'un processus consistant à développer certaines de ses caractéristiques jusqu'à un degré fortement discriminant. Cette «typicité »n'est pas acquise une fois pour toutes, elle évolue suivant la trajectoire que le chanteur se trace à l'intérieur du répertoire lyrique. Plutôt que d'employer un instrument donné par la nature, il s'agit d'en former un et de le modifier en fonction de l'usage souhaité. La construction de cet instrument mobilise une série d'acteurs et de pratiques institués. Les interactions entre le chanteur et ses partenaires forment le milieu de la construction sociale de l'ajustement : «... elle m'a beaucoup coaché non seulement musicalement mais aussi techniquement... elle a une grande connaissance de la technique vocale... et coache aussi disons... la vision du métier aussi. » (Baryton, $\mathrm{n}^{\circ} 8$ ) ; «... je vais auditionner aussi chez Caballé [...] qu'elle me dise... dans quel répertoire... qu'est-ce que je devrais faire maintenant... ou bien dans quel type de tournée... dans quel théâtre aller... vers qui auditionner.» (Baryton, $\mathrm{n}^{\circ} 8$ ) ; «... je dis aux personnes de s'adresser à l'agence [X]. Je dois dire que je les respecte vraiment parce que j'ai l'impression qu'ils se soucient aussi du parcours artistique. » (Soprano, $n^{\circ} 3$ ); «Bon, c'est important d'avoir un point d'ancrage, d'aller chez la même personne et de pouvoir se confier et de dire quel problème on a, etc. Mais je pense que c'est aussi important ces espèces de petits flashes qui peuvent venir de la part des collègues, même plus jeunes que soi.» (Soprano, $\mathrm{n}^{\circ} 3$ ) ; «...heureusement, il y a quelques grands directeurs d'opéras qui [...] se préoccupent vraiment et de l'opéra et de la carrière des chanteurs, c'est-à-dire qu'il y a une cohérence dans le travail, dans les exigences du metteur en scène, du chef d'orchestre, des chanteurs, etc. » (Ténor, $\left.n^{\circ} 11\right) ;$ «... un des très grands moments pour moi certainement ça a été la rencontre avec $[X]$, le metteur en scène, avec lequel je me suis entendu merveilleusement et qui m'a inclus dans des distributions fantastiques [...] qui $\mathrm{m}$ 'a introduit au fond vis-à-vis des directeurs des théâtres ou avec eux ou même imposé parfois, il a ... j'ai pu entrer un peu partout. » (Ténor, n ${ }^{\circ} 11$ ).

Le role-set du chanteur est extrêmement complexe et nous ne prétendons pas en rendre compte ici de façon extensive. Nous nous bornons à proposer un cadre où pourraient $s$ 'inscrire des analyses détaillées de ces interactions - entre

(suite de la note 23)

qu'elle enregistra mais n'exécuta jamais en public Hänsel et Gretel, Ariane à Naxos, Le Barbier de Bagdad, Les Contes d'Hoffmann, La femme avisée, Arabella, Troilus et Cressida... sans compter les opérettes (Elisabeth Schwarzkopf, 1983). 
chanteurs, professeurs, agents, directeurs de maisons d'opéra, chefs d'orchestre, metteurs en scène, etc. (24) - et de donner quelques-unes de leurs caractéristiques générales. Il ressort de notre enquête que les rôles tenus par les différents acteurs relèvent beaucoup plus des circonstances pratiques que des définitions « officielles». Du point de vue pratique, il y a des similitudes importantes entre les contributions de personnes occupant des positions très différentes sur le plan structural. Nous constatons également que ces rôles pratiques sont tenus simultanément et sur la durée d'une carrière par une multiplicité d'individus (y compris par l'artiste lui-même) : les chanteurs enchaînent ou cumulent les professeurs, multiplient les rapports avec différents employeurs, combinent fidélité à certains partenaires avec relations non récurrentes... Les ajustements se produisent à force de multiplier les occasions d'échange et les tensions semblent se dissoudre dans la fluidité des liens. Polyvalence, interchangeabilité et multiplicité sont les mots clés pour décrire 1'univers d'interactions entre les chanteurs et leurs partenaires professionnels.

La formation du chanteur se poursuit tout au long de sa carrière et se caractérise par l'emprunt d'une pluralité de voies - conservatoire, leçons privées, opéra studio, master classes -, par un long apprentissage portant sur différents aspects - solfège, technique vocale, diction, interprétation, expression corporelle, langues -, et par la mobilisation à des degrés plus ou moins «formels » de toutes sortes de «pédagogues » (le premier des rôles que nous avons repérés) - divers professeurs, coaches, accompagnateurs, répétiteurs, chefs d'orchestre, collègues... Ces mêmes acteurs réapparaissent lorsqu'il s'agit cette fois-ci non de doter l'élève de certaines compétences, mais de donner forme à son profil vocal et à son projet artistique. Le «guide» prévoit le développement de la voix, compose le répertoire, nuance les interprétations ; il est un «pédagogue », un chanteur célèbre dont la trajectoire sert de modèle ou qui est prêt à conseiller des jeunes talents, un chef d'orchestre qui prend sous son aile quelques protégés, un agent ou un directeur de maison d'opéra qui «construit » un artiste... Ces soins resteraient sans effet si d'autres (ou les mêmes) acteurs ne se préoccupaient pas de fournir au chanteur des occasions de travail effectives où employer ses compétences et mettre en œuvre ses projets. C'est avec les «managers »-agents, managers, directeurs de maisons d'opéra, chefs d'orchestre, metteurs en scène... - que les chanteurs négocient les engagements et les circonstances pratiques du processus d'ajustement (25).

(24) Il faudrait ajouter les représentants des maisons de disques, les critiques... et même les parents ou le conjoint du chanteur, personnages qui, dans cette profession où se réunissent vocation et entreprise familiale, jouent un rôle de premier ordre. Bien entendu, la situation d'un chanteur indépendant diffère de celle d'un salarié, mais même ce dernier a des activités artistiques en dehors de sa compagnie, ce qui élargit ses contacts professionnels; Towse (1993) donne des renseignements intéressants sur la situation professionnelle des chanteurs de troupe en Allemagne.

(25) Les divers professeurs bâtissent les compétences techniques, dramatiques, vocales, etc., de leurs élèves; les coaches offrent un enseignement avancé au cours duquel les chanteurs construisent leurs répertoires, préparent leurs auditions ou l'interprétation d'un rôle en entier. Aux États-Unis les chanteurs engagent, en plus 


\section{La détermination du rôle en fonction de la voix}

La forme d'un opéra est donnée par l'équipe artistique (chef d'orchestre, metteur en scène, directeur musical, chanteurs...) qui décide, entre autres, de la version à jouer, des passages à éliminer, de l'orchestration, du tempo. Rubato, crescendo et diminuendo, changements d'accents, coups d'archets, phrasés absents dans la partition mais nécessaires à son exécution, sont décidés lors des répétitions. Du point de vue vocal, la «production» actualise des rôles conçus dans le passé pour les artistes d'alors; l'équipe artistique va décider de leur conformité avec les voix actuelles jusqu'à aller parfois à l'encontre de ce qui a été fixé par le compositeur ou la tradition et changer le type vocal d'un personnage (26).

À entendre leurs déclarations, les chanteurs semblent considérer que la possibilité de concrétiser sur scène l'un ou l'autre des rôles «virtuels » dépend bien sûr d'un engagement, mais aussi pour une large part des circonstances de la «production»: «Par exemple, pour citer Les noces de Figaro, dans une grande maison, j'entends volume et avec une distribution très lourde, je chanterais Suzanne et donc dans une conception plus, pas légère, mais enfin différente, je chante la Comtesse, sans problème. » (Soprano, $n^{\circ} 5$ ). Les «managers »agents, représentants des maisons d'opéra, chefs d'orchestre, metteurs en scène... - négocient pour et avec le chanteur, au moment où se dessinent les lignes générales de la «production », le dimensionnement des rôles. Ces négociations portent sur divers aspects de la «production». Ainsi, avant d'accepter un engagement, les chanteurs cherchent-ils à connaître les noms de leurs collègues - «Quand Aix-en-Provence m'a offert Rinaldo,j'ai d'abord demandé quels seraient mes partenaires. L'amant d'Armida doit avoir l'instrument le plus sombre, le plus robuste : il était indispensable de réunir des ténors à la voix plus légère que la mienne » (27) -, ou à imposer leur choix quant à la salle - «Si se produire au Metropolitan Opera est une étape importante, conserver sa voix l'est encore plus. J'ai refusé de chanter Rosine et Idamante d'Idoménée dans un tel espace. En revanche, j'ai proposé Despina de Cosi fan tutte [...]. Je réserve Dorabella et Idamante à des salles de la taille du Capitole de Toulouse. » (28).

\section{(suite de la note 25)}

des services d'un agent, ceux d'un représentant qui s'occupe de la publicité, des relations publiques et des relations avec la presse. Obtenir des engagements, négocier les contrats, s'occuper de la facturation sont quelques-unes des tâches habituelles d'un agent, mais au sein de certaines agences, en plus de la «division générale», il y a une division de general management (engagements plus carrière), et/ou de secrétariat privé, offrant des services beaucoup plus larges. Aux États-Unis il y a une différence entre agent et manager, ce dernier s'efforçant de «créer » des occasions d'emploi mène une activité similaire à celle d'un impresario ou d'un organisateur de concerts européen. Démêler l'entrelacs d'acteurs et de pratiques, qui forme le monde lyrique, nécessiterait des études spécifiques pour chaque profession ; actuellement celles-ci sont presque inexistantes.

(26) L'exemple le plus probant a été donné par Karajan qui avait l'habitude d'attribuer des rôles importants à certaines voix plus légères qu'il n'était d'usage.

(27) Rockwell Blake, Opéra international (mai 1995, p. 17).

(28) Cecilia Bartoli, Le Monde de la musique (octobre 1992, p. 38). 
De même veulent-ils connaître la version retenue - «Quand Bologne, il y a quelques jours, m'a proposé Ariane auf Naxos, j'ai aussitôt demandé s'il s'agissait bien de la version de 1916 : il est hors de question que je me frotte à nouveau à celle de 1912. » (29) -, les airs à chanter, la langue des représentations, leur fréquence, etc. (30).

Une fois la «production» engagée, ces négociations - qui expriment les tensions lyriques - vont se poursuivre, cette fois-ci principalement entre les participants directs à la représentation. Notre enquête révèle une multitude de pratiques d'appropriation et de tactiques mises en place par les chanteurs, lors de la «production», pour donner au rôle des dimensions adéquates. Il s'agit de répondre dans un même mouvement aux critères d'excellence et de préservation vocales; de permettre au chanteur de faire une interprétation brillante - dans laquelle tout son talent sera déployé, qui surprendra par son originalité ou par sa «profondeur» - tout en préservant autant que possible l'aisance et la santé de sa voix : «... quand cette phrase se répète, liez le fa grave vers le haut, mais prenez une petite respiration rapide avant le deuxième fa aigu et attaquez à nouveau la note du haut. Cela donnera plus d'expression à la phrase et vous procurera plus de souffle pour la phrase suivante qui monte au sol bémol.» (Callas, 1991, p. 188). Au fur et à mesure que la date des représentations approche et que les lignes générales de la représentation sont fixées, les espaces d'indétermination semblent se réduire. Le remaniement du rôle devient plus subtil, il se fait par façonnage intime de la substance musicale et varie d'un jour à l'autre en fonction des circonstances de représentation - un rhume, un changement de partenaire, etc. Au cours des représentations, le chanteur adopte ainsi une démarche originale - une posture - composée de multiples gestes, autant de microdécisions par lesquelles il donne à la partition une forme, une couleur, un rythme particuliers.

\section{Postures lyriques. Une identité de base, trois dimensions fondamentales et un ensemble d'interactions}

Nous avons défini précédemment la posture comme la concrétisation pratique d'un rôle au cours d'une «production» (31). Mise en voix et mise en corps du texte, point d'intersection du chanteur et de son rôle, la posture prend forme dans la confluence des dispositions des acteurs, des positions offertes par la

(29) Sumi Jo, Opéra international (juilletaoût 1995 , p. 20).

(30) Le coup de force autant que la plus routinière des négociations semblent être plus ou moins à la portée du chanteur, comme des autres membres de la «production», en fonction de trois éléments : sa réputation, son «interchangeabilité », et le fait que son engagement soit ou non le fruit d'une cooptation. Même sans approfondir cet aspect, nos observations corroborent les résul- tats de certains travaux sur les systèmes de production artistique par projet (Chiapello, 1997 ; Faulkner et Anderson, 1987 ; Menger, 1989, 1991).

(31) Le terme de postures musiciennes désigne chez Mabru (1995) principalement la tenue de l'instrument (le violon) : le lien entre posture et projet musical est uniquement évoqué. Pavis (1983, pp. 85-86) utilise le terme d'attitude au sens brechtien de Haltung. 
«production» et des tensions qui agitent cette dernière. Elle est le creuset des interactions entre le chanteur et les autres membres de la «production». Ici, en plus de définir les dimensions des postures et de déterminer les pratiques qui leur sont attachées, nous allons repérer les traces de ces interactions de manière à situer l'interprétation dans son cadre d'occurrence.

Selon les sociologues interactionnistes précédemment cités, les accomplissements des rôles accumulés donnent forme à l'identité qui, à son tour, détermine la façon de jouer les rôles (32). Si nous envisageons les postures comme des cristallisations provisoires d'une définition de soi, il est intéressant d'examiner, avant toute autre considération, leur rapport avec l'identité.

\section{Identité et posture}

Les chanteurs se forgent une attitude vocale, musicale et dramatique lors de la «production». Cette configuration particulière du corps est obtenue à partir de l'encodage dans des actions et des signes d'un matériel de base: les autodéfinitions et hétérodéfinitions du chanteur en tant qu'individu, vocaliste, musicien, acteur ou professionnel. Alors que cette idée se laisse concevoir facilement, il est plus hasardeux de faire l'inventaire des « supports d'identité » lyriques et de déterminer la manière dont ils participent au façonnement de la posture. En dehors des compétences vocales, trois éléments paraissent plus particulièrement pertinents. Nous pensons tout d'abord à l'aspect physique du chanteur : sa taille, sa corpulence, son allure, la couleur de sa peau. La grande Jessye Norman se forge une image en accord avec ce qu'elle représente physiquement : impressionnante et majestueuse, traits par lesquels se caractérise chacune de ses présences scéniques. Indépendamment de la nature psychologique du personnage qu'il incarne, le chanteur va aussi «colorer » son interprétation par des traits de caractère qui, en tant qu'individu, lui sont propres. Elisabeth Schwarzkopf, en Maréchale, Donna Elvira ou la Comtesse de Capriccio, en imposait par son air aristocratique, fait de réserve et de maniérisme, là où d'autres artistes débordent de pathos, de passion et de sensualité. De mille façons, le chanteur signifie également sur les planches «qui il est en dehors», et notamment quelle position il occupe dans le monde professionnel. Occuper immanquablement le devant et le centre de la scène, prolonger ses airs avec des fioritures enchanteresses et apporter à chaque production ses propres costumes font partie des privilèges que réclament certaines prima donna ou autres stars.

C'est principalement avec le metteur en scène que le chanteur devra gérer le rapport entre «ce qu'il est» en dehors de la scène et «ce qu'il devient» lors de

(32) «J'ai soutenu que l'individu n'adhère pas au rôle qu'il trouve à sa disposition en maintenant tous ses autres Moi en suspens. [...] L'image qui ressort de l'individu est celle de quelqu'un qui synthétise et accommode, d'un jongleur et d'un conciliateur, qui remplit une fonction alors qu'il est apparemment engagé dans une autre ; il monte la garde à l'entrée de la tente mais fait entrer sous cape tous ses amis et parents. » (Goffman, 1961, p. 139) [traduit parl'auteur]. 
la «production» sur scène : «Je me mis en relation avec lui, racontais mes naufrages des années précédentes et lui demandais quelle conception il avait de Carmen, comment il imaginait le décor et la production en général. [...] Pour la première fois je rencontrais en Otti un metteur en scène qui bâtissait sa Carmen sur ma façon de bouger.» (Ludwig, 1996, p. 123). Aisée lorsque le rôle va à l'artiste «comme un gant», cette gestion est plus hasardeuse si son identité entre en contradiction avec les caractéristiques du personnage ou avec la conception dramatique du metteur en scène. Cela dit, certains se réjouissent du dédoublement et même le considèrent comme une des grandes satisfactions de la profession : «Le fait qu'il [Scarpia] apparaisse, somme toute, fort éloigné de ma propre personnalité accentuait encore davantage le bonheur de l'incarner, de s'en emparer. » (33). L'essentiel est de considérer la conformité ou la dissonance entre l'identité et le rôle autant comme le fruit des propriétés «inhérentes » aux deux entités que le résultat du processus d'ajustement. En fait, interprétation rime très souvent pour les chanteurs avec découverte. La gestion entre identité préalable et nouveau personnage se fait au profit d'un processus dans lequel l'artiste, d'une part, se découvre en tant qu'individu, expérimente un élargissement de soi, et d'autre part, découvre de «nouveaux » aspects du personnage, aspects qui font écho à certains traits de son identité (34).

\section{La construction, au sein des interactions, de l'ajustement sur les plans vocal, musical et dramatique}

La dimension vocale d'une posture lyrique se décrit en termes de tonalité, timbre, volume, tessiture, agilité, etc. La pratique du chant demande de la part de l'artiste autant d'efforts pour satisfaire les exigences de la partition que d'habileté pour contourner les difficultés qui lui sont insurmontables. À cette fin les artistes se permettent un certain nombre de libertés par rapport au texte musical. Une soprano ayant du mal à maîtriser son souffle fera fi de l'indication «piano» et restera pendant toute la partie dans le «forte». L'articulation de voyelles ouvertes pose problème dans les notes aiguës; au risque de nuire à la compréhension du texte, le ténor escamote certaines voyelles ou les modifie. Accélérer ou ralentir le tempo, retarder légèrement l'attaque d'une note, respirer lorsque la partition indique de passer sur une même respiration d'un son à un autre ; diverses libertés de ce genre sont à mettre en rapport avec la construction de l'ajustement sur le plan vocal (35). Si l'on s'accorde pour affirmer que le chant belcantiste correspond aux opéras préromantiques, alors que Verdi, les

(33) Gabriel Bacquier, Opéra international (mars 1995, p. 22). Lorsqu'on les interroge sur les satisfactions du métier, les comédiens étudiés par Menger (1997a) citent en premier lieu le plaisir attaché à la démultiplication et la connaissance de soi.

(34) Les interprétations de José Van Dam constituent d'excellents exemples de construction de la correspondance identité-rôles (Friche, 1988).

(35) Les accompagnateurs sont plus enclins à révéler ces astuces que les chanteurs, au point que parfois leurs déclarations ressemblent à un règlement de comptes ; voir Magiera (1990). 
opéras véristes ou Wagner requièrent un son plus «musclé », cette règle n'est pas absolue ; le chanteur adapte aussi ces conventions, pour autant qu'il en ait le choix, en fonction de sa condition physique, des sonorités qu'il souhaite obtenir ou du rôle à interpréter : «Quel a été votre rôle le plus dramatique ? [...] Le plus dramatique ? Desdemona d'Otello peut-être [...] Mais je l'ai chanté comme un rôle lyrique, simplement avec des accents dramatiques. On peut aussi aborder Butterfly comme un rôle dramatique, mais je ne l'ai pas fait. Là encore, je l'ai chanté en lyrique, avec des accents dramatiques. » (36).

Pour la construction de la dimension vocale, les artistes comptent sur l'appui des «pédagogues » extérieurs à la représentation et de ceux qu'ils trouvent sur place : un collègue, le chef de chant, le chef d'orchestre et ses assistants... Ceux-ci sont l'oreille externe qui contrôle la justesse de la voix ; ils localisent et expliquent les accrocs du chanteur et sont en mesure de proposer des «astuces » pour venir à bout des difficultés de la partition. Ces acteurs assurent également l'ajustement des prestations vocales, pour éviter qu'une voix «écrase » les autres ou porte atteinte à l'ensemble. Une fois devant le public, l'interlocuteur privilégié du chanteur pour ce qui est des questions vocales comme pour toute question d'ailleurs - est le chef d'orchestre. Il détient véritablement «dans ses mains » le pouvoir de modifier le tempo pour l'ajuster à la respiration du chanteur, de réduire le niveau sonore de l'orchestre pour permettre à l'artiste de «passer la rampe », de lui proposer toutes sortes d'altérations et d'interprétations de la partition ou, au contraire, d'exiger qu'il chante de façon «plus démonstrative » : «C'est très frustrant quand on a l'impression de devoir hurler et que le chef est là à faire des grands gestes. » (Soprano, $\mathrm{n}^{\circ} 3$ ). Bon nombre des différends entre les chanteurs et le chef d'orchestre portent sur ces questions ; ils sont significatifs des tensions que traverse l'opéra en tant que forme expressive hybride - chant, musique, théâtre - et des difficultés rencontrées par les artistes pour maintenir la cohésion entre ces différentes formes expressives.

Les compétences vocales d'un chanteur diffèrent de sa connaissance des conventions de l'interprétation propres à un compositeur, à un genre ou à un courant musical donnés ainsi que de son adresse à reproduire les sonorités appropriées. La musicalité du chanteur équivaut à la faculté de lire au-delà des notes, et de puiser dans l'histoire du chant, dans la tradition ou dans son propre «instinct musical» des indications sur la ligne mélodique, la ponctuation du phrasé ou l'ampleur du son qui convient à l'interprétation de tel ou tel rôle.

Les sources écrites - suggestions du compositeur, recueils de cadences et de passages - ou les enregistrements sonores aident le chanteur à rétablir une certaine atmosphère sonore sans commettre d'anachronismes. Au théâtre, les répétiteurs veillent à la cohérence stylistique des différentes prestations, et à leur

(36) Victoria de los Angeles, Opéra international (juin 1996, p. 14). Le bel canto vise une expression lyrique et un chant doux rarement lancé à pleine voix. Le chant dramatique aug- mente la brillance et la vaillance de la voix, mais demande de la part du chanteur une plus grande résistance physique. 
conformité avec les indications du chef d'orchestre. En vue d'une représentation, les chanteurs consultent chefs d'orchestres, coaches, répétiteurs, musicologues et anciens chanteurs réputés pour leur connaissance du rôle ou du répertoire en question : «J'appris mes rôles note après note avec Renato Pastorino qui m'a fait comprendre le vrai sens du style. $11 \mathrm{~m}$ 'expliqua que même si les notes et même les indications comme "avec passion" sont identiques dans toutes les partitions, la passion de Norma, par exemple, est différente de la passion de Santuzza ou de la Gioconda.» (37). Ces «pédagogues», en filiation plus ou moins directe avec les créateurs de la pièce, assument le rôle de dépositaires d'une certaine tradition d'interprétation musicale dont ils assurent la transmission. Sur ce plan, des désaccords surgissent entre les chanteurs, ou entre ceux-ci et le chef d'orchestre, sur le référent stylistique qui guidera l'interprétation. Le règlement de ces conflits façonne, de l'intérieur, la posture.

Depuis les premières décennies de ce siècle, qui marquent la clôture du répertoire, se fait sentir le besoin de productions qui, a défaut de montrer des nouveautés musicales, surprennent par la mise en scène (M. Harries et $\mathrm{S}$. Harries, 1986 ; Pleasants, 1989). Cette évolution a entraîné la montée en puissance des metteurs en scène et se traduit par une exigence dramatique accrue à l'égard des chanteurs. Les représentations actuelles accueillent difficilement, à moins qu'il ne s'agisse d'un virtuose réputé, une vedette au jeu statique, impressionnante par la taille et tout imbue de sa virtuosité vocale, comme on se représente communément le chanteur d'opéra. Bien au contraire, il est demandé à ce dernier de construire une image psychologique et physique du personnage que toute sa présence scénique doit s'évertuer à transmettre au public. Au cours des répétitions, on assiste à des négociations entre les divers participants à la «production », notamment entre le chanteur, le chef d'orchestre et le metteur en scène, concernant la dimension dramatique. Les chanteurs rechignent à trahir d'une façon ou d'une autre leur identité - physique, psychologique, professionnelle ou à changer trop radicalement leur conception du rôle. Plus concrètement, ils demanderont au metteur en scène que la construction théâtrale de leur posture ne se fasse pas au prix d'un sacrifice de la dimension vocale : les mouvements brusques et les distorsions du corps sont à éviter lors des passages difficiles ; au moment des arias, on assurera la frontalité entre chef et chanteur ; les décors ne doivent pas absorber le son ni les costumes oppresser la poitrine ou couvrir la tête, etc.

Nous découvrons un subtil jeu d'interrelations entre les composantes de la posture - le côté vocal dépend de la vision dramatique du rôle autant qu'il est subordonné à la dimension musicale - et une recherche constante de la part des acteurs pour les équilibrer, les accorder, compenser la faiblesse d'une dimension en donnant plus d'importance aux autres, etc. Les membres de la «production» n'hésitent pas à faire jouer l'indétermination de la partition et du livret, voire à forcer l'une et l'autre en vue d'harmoniser leurs contributions. Le chef d'orchestre, actif à la fois dans la fosse et sur le plateau, est le rassembleur d'entités

(37) Ghena Dimitrova dans Matheopoulos (1992, p. 78) [traduit par l'auteur]. 
distinctes - le chœur, l'orchestre, les solistes - et le garant de la symbiose de ces trois dimensions. Le travail d'ajustement devient particulièrement visible lorsque la voix du chanteur diffère substantiellement de celle pressentie habituellement pour le rôle en question. Prenons par exemple le cas d'une soprano lyrique à qui le chef d'orchestre propose d'incarner Salomé - rôle très dramatique, chanté normalement par de grandes voix à même de faire ressortir le caractère violent de celle qui commande l'exécution du Baptiste. Le chef d'orchestre connu par ces interprétations «immaculées » et «cristallines » promet à l'artiste de diriger l'orchestre de Strauss tel un ensemble de musique de chambre. Le metteur en scène «voit» dans Salomé une adolescente obstinée en quête d'un idéal de pureté, frustrée et anéantie par le refus que lui oppose le prophète. Dans la scène finale, aucune trace de déchaînement luxurieux, mais la présentation d'un désespoir contenu. Grâce à cette interprétation du rôle, le son orchestral et vocal peut se montrer tel que le souhaitait le chef et qu'il convenait au chanteur : allégé, raffiné et délicat (38). Ces subtils modifications et ajustements font plus qu'assurer l'interprétation - éventuellement la cohérence du personnage et de la mise en scène -, ils sont à la base de l'individualisation des prestations autant que des identités artistiques (39).

\section{Tensions lyriques}

À plusieurs reprises, nous avons fait référence au caractère mouvant de la posture lyrique. Celle-ci résulte d'un travail d'ajustement entre les attentes, les besoins, les intérêts et les compétences - multiples et contradictoires - des différents membres de la «production », qui ne se révèlent que de façon progressive et se déterminent réciproquement. Cette construction en commun de la posture se présente autant sous le mode de la collaboration que du conflit. Le corps du chanteur s'est révélé tiraillé non de façon erratique mais entre des pôles opposés, traversé par ce que nous avons appelé les tensions lyriques, qui exerceraient leur force sur les trois dimensions de la posture - vocale, musicale et dramatique. C'est ainsi que les postures se déplacent sur une série d'axes dynamiques en fonction des interactions que les chanteurs entretiennent entre eux, avec les chefs, les metteurs en scène... Les axes dessinent les potentialités de l'interprétation. Les positions sont interchangeables, susceptibles d'être occupées au gré des circonstances par chaque membre de la production : un chef d'orchestre puriste affronte un chanteur plein de fantaisie, un ténor déchaîné

(38) Sur les différentes interprétations musicales, vocales et dramatiques de Salomé, voir Matheopoulos (1992).

(39) «C'était effectivement mon point fort la musicalité. Beaucoup plus que la puissance. » (Baryton, $n^{\circ} 9$ ). «Il faut apprendre à maîtriser totalement son corps. Ainsi - au moment critique - on sait comment détourner subtilement l'atten- tion et compenser les défaillances vocales par l'interprétation, en captivant les spectateurs par la force de sa personnalité. " (Visnevskaja, 1985 , p. 51). «Toute ma vie, j'ai voulu être une grande artiste. Je n'en suis pas une. Je suis une chanteuse avec une belle voix. » (Montserrat Caballé dans Mathepoulos, 1992, p. 72) [traduit par l'auteur]. 
malmène vocalement sa partenaire à moins que cela ne soit le contraire. Les extrêmes des axes correspondent à des postures intenables dans lesquelles le chanteur s'annule ou la performance lyrique devient hautement problématique.

Ces tensions agitent toute action collective : les acteurs ont forcément à déterminer le degré auquel leur participation sera innovatrice, et à quel point ils comptent s'investir et collaborer à l'action commune (40). Lors d'une «production » d'opéra, ces tensions prennent une forme particulière relative aux caractéristiques du travail lyrique : l'opéra est réactualisation du passé, performance physique et imbrication de contributions diverses.

\section{Entre reproduction et innovation}

La «production» lyrique est un cours d'action collective qui mène à la construction d'une forme musicale originale à partir de certains éléments hérités du passé. Mettre en scène un opéra revient à proposer une lecture de l'œuvre et de ses réceptions, à se situer par rapport à la tradition. Les participants à la «production » sont inévitablement pris dans un jeu de renvois avec les interprétations antérieures et ont à gérer un rapport à l'histoire : «Je respecte trop Bellini et les grandes interprètes du rôle qui m'ont précédée. Bien entendu, je ne ferai jamais une Norma comme celle de Callas. Je suis une chanteuse différente et une personne différente. Mais sa Norma était une Norma dont il fallait tenir compte. » (41). Chaque nouvelle interprétation incarne le conflit entre restituer, perpétuer les traditions interprétatives des formes instituées, et parvenir à les dépasser pour produire du nouveau et préserver la singularité de la situation.

L'antagonisme reproduction-innovation est d'une grande complexité en ceci que les acteurs vouent fidélité simultanément à plusieurs entités parfois contradictoires et de nature composite de surcroît. Les chanteurs mentionnent principalement trois références préexistant à la «production», par rapport auxquelles ils doivent, dans une relation de reproduction ou d'innovation, donner forme à leur présence scénique. Il s'agit des indications du compositeur (notamment la partition), de la tradition musicale et interprétative et de l'identité propre à l'artiste. Ces entités fonctionnent en tant que guides pour l'action, outils de définition d'une situation nouvelle, ressources pour réduire tout élément novateur à un cas type. Lorsque des rapports d'opposition lient les entités - par exemple une norme interprétative contraire à la notation musicale - , la possibilité s'ouvre pour le chanteur de revendiquer l'authenticité de sa démarche limitée en fait à un seul plan -, tout en s'éloignant d'une attitude trop respectueuse. Un exemple nous est donné dans l'interprétation de Norma par Maria Callas, un rôle traditionnellement exécuté par une voix plus claire que celle de

(40) Notre analyse des tensions lyriques definition du cadre, engagement, foyers d'attention, interaction éparpillée, indications scéniques et operations de réalignement - doit beaucoup à la lecture de Goffman.

(41) June Anderson dans Matheopoulos (1992, pp. 30-31) [traduit par l'auteur]. 
sa rivale Adalgisa. En incarnant une prêtresse à la voix riche, «...à la fois sombre, agile, élégiaque et tragique » (Moindrot, 1993b, p. 38), Callas restaure l'équilibre entre les voix et se présente comme le fidèle serviteur des intentions du compositeur.

Nous avons vu que les indications du compositeur, ainsi que la tradition, entités indéterminées, plurielles ou contradictoires, ne constituent pas en ellesmêmes des modèles suffisamment précis sur lesquels les membres de la «production » peuvent calquer leur interprétation. Il y a façonnement, au cours de la «production » et à des fins pratiques, de deux référents ad hoc, « la partition» et «la tradition». La démarche des acteurs peut être décomposée, à des fins analytiques, en deux moments en réalité souvent accomplis simultanément. Tout d'abord, élaboration et «monstration», lors du travail en commun, des références en fonction desquelles, dans un deuxième temps, ils établissent un rapport qui oscille entre les pôles de la reproduction et de la rupture. La constitution et la caractérisation des référents vont de pair avec toute digression ou conflit sur la prétendue fidélité ou transgression de la posture. Échecs et mécontentements relèvent très souvent d'une difficulté à s'entendre sur le référant à partager et sur le type de rapport à établir avec celui-ci. Une situation de conflit relativement fréquente se produit lorsque le chef, mû par des ambitions puristes, se propose de «nettoyer» l'œuvre - des airs de bravoure par exemple - et retire ainsi au chanteur des possibilités de se distinguer.

Il y a, au cœur de l'interprétation, ce dialogue avec le passé, dialogue dont ce dernier ressort concrétisé, façonné et prêt à l'emploi. Néanmoins, les références ne sont pas uniquement historiques ou de nature identitaire. À mesure que le projet lyrique prend forme, de nouveaux «éléments tuteurs » se dégagent du cours d'action sur lequel ils vont exercer une influence similaire à celle du texte musical, de la tradition ou de l'identité. Une certaine forme d'expression corporelle ou vocale, des consignes métronomiques acquièrent ainsi à leur tour le poids de la norme. Leur établissement, soumis à la tension « reproductioninnovation", constitue un des enjeux centraux des interactions et façonne profondément l'interprétation.

\section{L'art de la mesure}

L'antinomie «réserve-abandon» se retrouve dans de nombreuses descriptions des activités artistiques, lesquelles s'assimileraient à l'engagement, au don de soi ou au risque, et s'opposeraient à une attitude de contrôle, de retrait ou de calcul. En passant des idéaux à la réalité des pratiques, on constate que les acteurs ont à formuler en toutes circonstances à la fois leur façon de s'engager dans un cours d'action - ce que nous avons appelé posture - et leur degré d'engagement. Dans le cas de la production lyrique, la question de la mesure de l'engagement vocal se pose de façon particulièrement pertinente. Un mauvais usage de la voix, notamment l'inadéquation entre les compétences du chanteur et les exigences du rôle, exigerait pour l'artiste de forcer son instrument, ce qui pourrait entraîner des altérations vocales graves (vibratos irréguliers, enrouements, aphonies), 
voire irréversibles. Comme il a été montré, la préservation de la voix est un principe régissant toute la trajectoire d'un chanteur, en rapport avec la notion de compétences vocales dont nous avons déjà relevé la nature fluctuante, construite en cours d'action. Il nous reste à comprendre comment la tension résultant de ces deux contraintes - préserver la voix et donner de la voix - habite la posture et est gérée lors de la «production».

«Préserver la voix », l'injonction s'applique autant pendant le temps de repos, les répétitions que lorsque l'artiste se trouve devant le public. Elle entre en contradiction, pour ce qui est de l'interprétation, avec le besoin de préparer, d'entraîner la voix et de «mettre le rôle dans la gorge». Lors des répétitions, certains artistes se limitent à «marquer» leurs airs, d'autres préfèrent chanter la partie en entier et à pleine voix. Avant de monter sur scène, tel chanteur repasse dans sa loge les passages les plus difficiles de son rôle, alors que sa partenaire refuse de laisser s'échapper la moindre vocalise. «...[L]'habitude est prise au palais Garnier : chaque matin, les assistants-metteurs en scène appellent tous les solistes pour s'assurer qu'ils sont en forme et qu'ils chanteront le soir. » (Sarazin, 1988, p. 120). La gestion des forces vocales est à rapprocher de nombreuses pratiques ou situations courantes dans le monde lyrique : laryngologue de garde en coulisses, engagement de covers pour les rôles principaux, alternance de deux sopranos dans le rôle-titre au cours d'une série de représentations, ajournement de la première demandé par une diva, remplacement du ténor par un autre au début du deuxième acte, etc.

La représentation, sorte d'épreuve d'endurance, demande de la part du chanteur un contrôle important de ses forces, qu'il lui faut employer en fonction des difficultés de la partie. Doser les accents dramatiques, qui demandent au chanteur de «pousser» la voix, en adoptant autant que possible une émission lyrique ; ralentir un tempo trop pressant ; voler ici et là de petites respirations ; retarder quelque peu l'attaque d'une note difficile constituent des stratagèmes propres à une utilisation rationnelle de la voix : «C'est quoi le faire [chanter] d'une manière intelligente ? De respecter sa voix. C'est-à-dire de pas vouloir forcément avoir... grossir sa voix ou avoir plus de volume, mais de savoir faire des pianos, pour donner l'impression qu'on peut faire un crescendo ou des choses comme ça. Oui, respecter ses possibilités et donner l'illusion qu'on a une grande voix... » (Soprano, $n^{\circ} 3$ ). Cette attitude ne convainc évidemment pas les partisans de l'abandon et de l'engagement total, elle étonne ou paraît extrêmement froide et rationnelle aux chanteurs - notamment aux jeunes - qui avouent fonctionner «beaucoup à l'enthousiasme, à l'instinct». À certains moments, même le chanteur le plus prévoyant peut se sentir assez sûr pour quitter son attitude de réserve et rechercher la difficulté supplémentaire : il bisse un air de bravoure, improvise une cadence éblouissante, trille un si naturel, «tient» interminablement une note, multiplie les sonorités de poitrine, etc.

L'engagement est une matière permanente de conflit entre les chanteurs lorsque ceux-ci peinent à ajuster leurs voix : «Vous aurez besoin de beaucoup de souffle non seulement pour le crescendo qu'il faut faire avec le ténor sur sol, mais parce qu'il va probablement, j'ai le regret de le dire, se mettre à brailler. Pardonnez-moi l'expression, mais elle n'est que trop exacte.» (Callas, 1991, 
p. 254) ; entre les chanteurs et les chefs d'orchestre qui ne parviennent pas à «retenir» l'orchestre, imposent un diapason anormalement haut, des cordes métalliques qui rivalisent avec les voix, ou des salles aux proportions et à la configuration plus ajustées à l'orchestre qu'aux voix humaines (42). Les chanteurs blâment le metteur en scène qui leur demande davantage d'expressivité vocale ou de retenue qu'ils n'ont l'habitude d'en donner, ou qui leur impose des mises en scène contraignantes du point de vue physique. Et en dernier lieu, la question de l'engagement oppose le public, notamment ses membres les plus bruyants, aux chanteurs en panne de vaillance vocale (43).

\section{Dissociation ou cohésion}

En janvier 1981, Pavarotti se trouve au Royal Opera House de Londres, pour répéter Le Bal masqué. Il exprime au chef Bernard Haitink son souhait de faire un retenuto - qu'il dit faire partie de la meilleure tradition - au cours du trio qui suit le duo d'amour à l'acte II ; le chef d'orchestre s'y oppose. Arrive la première représentation : ainsi qu'il l'avait annoncé, le ténor s'attarde sur sa phrase, le chef refuse de prolonger l'accompagnement et l'orchestre poursuit de l'avant. Comme les entrées vocales des autres chanteurs - la soprano et le baryton - sont décalées, ceux-ci restent muets. Pavarotti finit sa phrase et quitte la scène ; sans le ténor plus de suite possible à l'action, le rideau tombe (Pullen et Taylor, 1996, p. 253). Cette anecdote rend compte d'un risque inhérent à toute action collective : la désagrégation du groupe suite à un désaccord sur la façon d'interagir. La représentation suppose la collaboration d'un nombre important de personnes, aux intérêts souvent contradictoires, entretenant de forts liens de dépendance réciproque. La cohésion naît lorsque les participants, par de subtils réglages interindividuels, ajustent leur performance à celle de leurs collègues et jouent en tenant compte de l'interaction de leur rôle avec le reste de la «production »: «Dans Dorabella, ma principale préoccupation est de me rapprocher de Fiordiligi, sur le double plan vocal et interprétatif. Quelle que soit ma partenaire, je cherche toujours à suivre, à opérer l'osmose la plus parfaite entre nous. » (44). Le maintien de la coopération demande aux participants de s'en tenir au comportement convenu au préalable, de se faire confiance et de se rendre mutuellement visible le sens de leurs actions. Les indications scéniques mutuelles abondent ainsi que les signes de lien, toute entorse à la solidarité étant suivie de représailles ou d'opérations de réalignement.

La représentation apparaît comme un assemblage d'individualités et de dimensions qui risquent de s'éloigner les unes des autres. Ce caractère «déta-

(42) Sur ces différents aspects, voir BovierLapierre (1988), Chuilon (1994) et Pleasants (1989). Une solution (extrême) aux difficultés des chanteurs consiste à les doter de micros.

(43) Nous ne disposons que de très peu de renseignements sur la réception de l'opéra, et pourtant le public participe de façon directe (et particulièrement bruyante lorsqu'il s'agit des membres des «cliques » et des «claques»), sinon à la «production», du moins à la représentation.

(44) Susanne Mentzer, Opera international (avril 1997, p. 6). 
chable» des prestations permet d'envisager facilement une «production » organisée en fonction d'un seul protagoniste, ou une posture favorisant un seul aspect de l'œuvre. L'attitude de la diva correspond au premier type de dissociation. Elle nous renseigne sur la caractéristique qu'a l'opéra d'être à la fois le produit d'un collectif et une suite de prestations individuelles qui se suffisent largement à elles-mêmes pour attirer le public. La chanteuse qui se conduit en diva lâche pour ainsi dire l'ensemble. Point de sentiment «collégial » chez elle, elle tente d'organiser le tout de façon à mettre en évidence ses seules qualités ou à cacher ses faiblesses : «... je suivis mon petit bonhomme de chemin jusqu'à ce fichu si bémol aigu, à la fin de l'air, que je filai délibérément pianissimo assez longtemps pour que le public crût que j'en resterais là. Au dernier moment, cependant, je l'enflai brusquement [...] Une fois que j'eus lâché la note et fus tombée en pleurs aux pieds de Calaf, un rugissement s'éleva du public et la représentation fut interrompue pendant un bon moment.» (Visnevskaja, 1985, p. 327). La diva refuse d'harmoniser sa voix avec celles de ses partenaires, se sépare du groupe pour se mettre en avant, ménage au terme de sa prestation le ritardando imperceptible qui déclenche des applaudissements, bisse son air, sort saluer seule devant le rideau, etc. Dans son attitude il y a déplacement du foyer d'attention, puisqu'elle néglige le contact avec le plateau pour s'adresser en premier lieu au parterre. Elle joue, en tant qu'individu, directement pour le public, et si elle se sert de l'ensemble, elle refuse par contre d'en être la médiatrice.

Dans le contexte de la «production», se désolidariser veut dire aussi privilégier une seule dimension de la posture au détriment de l'opéra comme hybridation de formes expressives diverses - théâtre, chant, musique, danse. Parce que la posture exprime les interactions du chef d'orchestre, du metteur en scène ou des musiciens, lorsque le chanteur délaisse une dimension, il perd d'une certaine manière le contact avec une partie de ses collègues. Un vocaliste virtuose néglige les indications du metteur en scène et, immobile devant la rampe, fait exhibition de ses talents vocaux sans faire grand cas du texte. C'est l'image même de la controverse qui agite le genre depuis la création de l'opéra - «prima la musica o prima le parole »-en train de s'incarner et de se réactualiser dans une posture lyrique, dans les relations qui la lient à la «production» et dans les tensions qui la traversent.

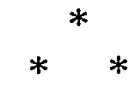

Nous voici au terme d'un parcours que nous avions entamé avec l'objectif de bâtir un cadre d'analyse capable d'appréhender ce qui nous semble être le cœur même de l'activité du soliste d'opéra : l'interprétation lyrique. Au cours de cette recherche, nous avons été amené à évoquer les interactions entre le chanteur et son entourage, la construction des compétences lyriques, l'indétermination des partitions musicales et leur détermination lors de la «production », les dimensions des postures et les tensions qui les façonnent; autant d'aspects que nous avons articulés autour d'une problématique centrale : le travail interprétatif 
lyrique comme ajustement dynamique et détermination réciproque, dans un contexte de «production", des compétences et des rôles (45). L'ajustement voix-rôle se présente comme une problématique à la fois tout à fait spécifique et centrale à l'interprétation lyrique, assez générale pour nous permettre d'appréhender un grand nombre de situations lyriques fort diverses, et susceptible d'être affinée pour servir à l'analyse d'une situation interprétative particulière. Les interprétations résultent d'un processus au cours duquel se succèdent les négociations, coopèrent et rivalisent les différents participants à la «production ». La thèse de 1'ajustement permet de rendre compte de façon dynamique de l'interaction de ces acteurs et des tensions qui façonnent les échanges.

Le point focal de notre réflexion a porté sur l'interprétation lyrique au cours de la «production». Néanmoins, notre recherche livre quelques éléments pour l'étude des identités artistiques et des carrières professionnelles, ce qui, en dehors des situations concrètes de travail, a constitué un objet d'étude majeur des sociologues interactionnistes (Barley, 1989). Leur conception de la carrière comme succession de rôles et de vécus subjectifs des rôles, ainsi que comme formulation et modification d'une identité professionnelle, nous semble fort appropriée au cas des solistes. La façon qu'un chanteur a, au cours de sa carrière, de doser les composantes de ses postures et sa manière de gérer les tensions inhérentes à l'interprétation pourrait constituer des principes de différenciation des identités et de caractérisation des trajectoires artistiques et professionnelles.

Qu'il s'agisse de rendre compte de la composition d'un répertoire, de la préparation d'une distribution ou d'une défaillance vocale, le sociologue s'entendra répéter par les chanteurs, les professeurs de chant ou les agents qu'un interprète doit «prendre uniquement les rôles qui correspondent à sa voix». Alors que cette expression laisse supposer deux entités aux caractéristiques parfaitement connues, stables et antérieures au moment du choix, nous avons montré leur caractère fortement fluctuant. Dans son ensemble notre travail invite à reconsidérer la nature des conventions et des catégories propres au monde artistique. L'idée est connue : des formes de pensée communes acquises par socialisation, en vigueur dans une discipline et une époque déterminées, procurent les bases de la participation du monde de l'art à une action collective (Becker, 1988). Nos observations sur la construction à la fois de la voix et des «exigences » des rôles éclairent une des caractéristiques de tels référents collectifs, celle de se remplir d'un sens nouveau lors de leur application, tout en préservant leur caractère stable et rassembleur à l'égard de la communauté qui s'y réfère.

Pour répondre aux interrogations de la sociologie de l'art, nous avons associé les acquis de la sociologie interactionniste du travail à ceux de la théorie de

(45) Notre exploration des pratiques lyriques voudrait rendre un peu moins mystérieux le processus décrit par Bourdieu : «Si le poste fait l'habitus (plus ou moins complètement), l'habitus qui est d'avance (plus ou moins complète- ment) fait au poste (du fait des mécanismes déterminant la vocation et la cooptation) et fait pour le poste, contribue à faire le poste. » (1980, p. 211). 
l'action. Deux principes ont guidé la formulation de notre démarche, d'une part, explorer «la pâte » même du travail lyrique sans nous perdre dans le particulier mais en faisant ressortir les constituantes essentielles et la dynamique générale des processus interprétatifs, d'autre part, construire un cadre d'analyse qui tiendrait compte à la fois de la variabilité des postures et du poids des éléments donnés à l'avance - la partition, la tradition, les rôles, etc. - en insérant ces derniers au cœur même de l'interprétation.

Esther GONZÁLEZ MARTÍNEZ

Institut des Sciences Sociales et Pédagogiques BFSH2-Université de Lausanne CH-1015 Lausanne

Esther.GonzalezMartinez@issp.unil.ch

\begin{abstract}
ANNEXES
I - Méthodologie

Ce travail a démarré dans le cadre d'une recherche collective : "Créativité et professionnalité chez les musiciens-interprètes en Suisse » et «Créativité et professionnalité. Parcours individuels et marchés du travail des musiciens » (Subsides FNRS n 12-32449.91 et n"12-39667.93-1992-1995), dont les résultats ont fait l'objet de plusieurs publications : Willener (1997a, 1997b).

En tant qu'assistante de recherche du professeur Alfred Willener et de Roberta Shapiro, l'auteur a pu bénéficier de l'accès aux documents et matériaux empiriques recueillis lors de ces enquêtes : plus d'une centaine d'entretiens semi-directifs (auprès de chefs, musiciens, enseignants - dont six professeurs de chant -, responsables administratifs et agents artistiques - dont quatre agents lyriques), statistiques nationales, observations de situations professionnelles - auditions, répétitions et représentations -, biographies et interviews publiés, rapports administratifs et documentation spécialisée. Rassemblés, ces matériaux donnent un aperçu général du monde artistique dans lequel évoluent les chanteurs lyriques.

Pour ce qui est du travail d'interprétation, notre article prend appui sur un corpus de douze entretiens semi-directifs avec des chanteurs lyriques suisses. Nous avons identifié chaque artiste par un numéro et trois caractéristiques : voix, stade de sa carrière et notoriété. 1) Alto, final, nationale ; 2) Mezzo, débutant, nationale ; 3) Soprano, débutante, européenne ; 4) Soprano, mi-carrière, nationale ; 5) Soprano, mi-carrière, européenne ; 6) Soprano, à la retraite, nationale ; 7) Baryton, débutant, nationale ; 8) Baryton, débutant, nationale ; 9) Baryton, mi-carrière, nationale ; 10) Ténor, mi-carrière, nationale ; 11) Ténor, à la retraite, mondiale ; 12) Ténor, à la retraite, mondiale. Au cours de ces entretiens, il a été demandé aux artistes de retracer leur trajectoire professionnelle et de parler de certains aspects de leur pratique artistique : choix et préparation des rôles, caractéristiques du dernier spectacle, relations avec les collègues, projets professionnels, etc. Nous avons également analysé un corpus de données publiées composé de tous les entretiens (plus d'une centaine) avec des chanteurs publiés par les revues Opera et Opéra international entre 1995 et 1997, et de 26 ouvrages à caractère biographique ou autobiographique sur des chanteurs célèbres de l'après-guerre à nos jours, choisis à partir des bibliographies de Cowden (1994) et de Farkas (1985).
\end{abstract}




\section{Revue française de sociologie}

\section{II - Définitions}

Caractérisation du son vocal (46). On s'accorde à caractériser les voix selon trois paramètres acoustiques : 1) l'intensité est fonction de la valeur de la pression qui s'exerce sous la glotte, 2) la hauteur est directement en relation avec la fréquence de vibration des cordes vocales, 3) le timbre est le résultat d'une modulation du son laryngé à travers les cavités de résonance : le pharynx, la cavité buccale, les fosses nasales.

Classification des voix. Les voix sont classées selon leur tessiture, qui désigne l'échelle des sons qu'un chanteur parvient à émettre aisément. La répartition la plus courante comprend, de la plus grave à la plus aiguë, trois voix féminines : contralto, mezzo-soprano et soprano, et trois voix masculines : basse, baryton et ténor. Toutes les voix d'une même catégorie ne correspondant pas de façon identique à un même intervalle, il existe donc des sous-catégories dérivées des types vocaux de base. Par exemple, parmi les sopranos (do3-do5) on trouvera le soprano léger (do3-mi ou fa5), lyrique-léger (do3-ré5), lyrique (do3-do5) et dramatique (la2-do5). Mais les voix sont classées également par rapport à d'autres facteurs comme la qualité de l'émission, le timbre ou l'agilité du chanteur à égrener ses notes (le colorature). En outre, les subdivisions et les réalités vocales auxquelles les classifications font référence changent selon les pays, les époques, les répertoires et même les écoles de chant. En définitive, on peut dire que les termes employés désignent plus souvent des types d'emplois que des voix différentes.

Les registres de la voix et le passage. On appelle registre un ensemble de sons émis par une même mécanique vibratoire : 1) le registre de poitrine produit les sons graves de la voix, le timbre est riche en harmoniques, les cordes vocales sont relâchées, les vibrations amples, 2) le registre de tête produit les sons aigus, de faible intensité, les cordes vocales sont tendues, les vibrations de faible amplitude. Au moment du passage du registre de poitrine au registre de tête, le timbre devient plus doux, la voix instable et moins puissante. Pour obtenir une plus grande homogénéité, le chanteur lyrique tente de mélanger les deux registres et de faire en sorte que le passage ne s'entende pas. L'émission de notes aiguës en registre de poitrine exige du chanteur un effort supplémentaire.

(46) Pour ces notes techniques nous nous référons à Cross (1991) et à Rosenthal et Warrack (1995).

\section{RÉFÉRENCES BIBLIOGRAPHIQUES}

Adorno T. W., 1994. - Introduction à la sociologie de la musique : douze conférences théoriques, Genève, Contrechamps.

Alford R. R., Szanto A., 1996. - «Orpheus wounded : the experience of pain in the professional worlds of the piano ", Theory and society, 25, 1, pp. 1-44.

Barley S. R., 1989. - «Careers, identities, and institutions : the legacy of the Chicago school of sociology » dans M. B. Arthur, D. T. Hall, B. S. Lawrence (eds.), Handbook of career theory, Cambridge, Cambridge University Press, pp. 41-65.

Becker H. S., 1985. - Outsiders : études de la sociologie de la déviance, Paris, Métailié.

- 1988. - Les mondes de l'art, Paris, Flammarion.

Bianconi L., Pestelli G. (éds.), 1992. - Histoire de l'opéra italien, Liège, Mardaga.

Bourdieu P., 1980. - Questions de sociologie, Paris, Éditions de Minuit.

Bovier-Lapierre B., 1988. - Opéras : faut-il fermer les maisons de plaisir? Nancy, Presses Universitaires de Nancy.

Callas M., 1991. - Leçons de chant : master classes à la Julliard School 1971-1972, Paris, Fayard.

Celletti R., 1987. - Histoire du bel canto, Paris, Fayard. 
Chiapello E., 1997. - «Les organisations et le travail artistique sont-ils contrôlables ?», Réseaux, 86, pp. 77-113.

Christiansen R., 1984. - Prima donna : a history, London, The Bodley Head.

Chuilon J., 1994. - Opéra, opinions, Paris, Romillat.

Corbin J. M., Strauss A. L., 1993. - « The articulation of work through interaction », The sociological quarterly, 34, 1, pp. 71-83.

Cottereau A., 1994. - « Théories de l'action et notion de travail : note sur quelques difficultés et quelques perspectives », Sociologie du travail, 36, HS, pp. 73-89.

Cowden R. H., 1994. - Classical singers of the opera and recital stages : a bibliography of biographical materials, Westport, Greenwood Press.

Cross R. (éd.), 1991. - La voix dévoilée : actualités scientifiques sur la voix chantée, Paris, Romillat.

Elisabeth Schwarzkopf, 1983. - Paris, L'Avant-Scène Opéra.

Escal F., 1991. - «Le corps social du musicien», International review of the aesthetics and sociology of music, 22, 2, pp. 165-186.

Farkas A., 1985. - Opera and concert singers : an annotated international bibliography of books and pamphlets, New York, Garland.

Faulkner R. R., Anderson A. B., 1987. - « Short-term projects and emergent careers : evidence from Hollywood », American journal of sociology, 92, 4, pp. 879-909.

Friche M., 1988. - José Van Dam, Paris, Buchet/Chastel.

Future (The) of opera, 1986. - Lanham, University Press of America.

Goffman E., 1961. - Encounters : two studies in the sociology of interaction, New York, The BobbsMerrill Company.

- 1968. - Asiles : études sur la condition sociale des malades mentaux et autres reclus, Paris, Éditions de Minuit.

- 1973. - La mise en scène de la vie quotidienne, Paris, Éditions de Minuit.

- 1974. - Les rites d'interaction, Paris, Éditions de Minuit.

- 1975. - Stigmate : les usages sociaux des handicaps, Paris, Éditions de Minuit

- 1987. - Façons de parler, Paris, Éditions de Minuit.

González Martínez E., 1998. - «L'art de la mesure : sur le rapport entre choix du répertoire et défaillances vocales des chanteurs lyriques» dans $\mathbf{R}$. Shapiro (éd.), Créativité et professionnalité chez les musiciens-interprètes en Suisse : parcours individuels et marchés du travail des musiciens, Rapport final de recherche, Lausanne, Institut de sociologie des communications de masse.

Harries M., Harries S., 1986. - Opera today, London, Michael Joseph.

Hartland N. G., 1994. - « Goffman's attitude and social analysis », Human studies, 17, 2, pp. 251-266.

Heinich N., 1995. - «Façons d'être écrivain : l'identité professionnelle en régime de singularité », Revue française de sociologie, 36, 3, pp. 499-524.

Hennion A., 1993. - La passion musicale : une sociologie de la médiation, Paris, Métailié.

Hughes E. C., 1958. - Men and their work, Glencoe, Free Press.

Jauss H. R., 1978. - Pour une esthétique de la réception, Paris, Gallimard.

Kamerman J. W., Martorella R. (eds.), 1983. - Performers and performances : the social organisation of artistic work, Massachusetts, Bergin \& Garvey Publishers.

Lacombe H., 1997. - Les voies de l'opéra français au XIX' siècle, Paris, Fayard.

Lehmann B., 1995. - «L'envers de l'harmonie», Actes de la recherche en sciences sociales, 110 , pp. 3-21

Leibowitz R., 1997. - Histoire de l'opéra, Paris, Buchet/Chastel.

Ludwig C., 1996. - Ma voix et moi, Paris, Les Belles Lettres.

Mabru L., 1995. - «Des postures musiciennes », Ethnologie française, 25, 4, pp. 591-607.

Magiera L.,1990. - Mirella Freni : metodo et mito, Milano, Ricordi.

Major N., 1994. - Joan Sutherland : the authorized biography, London, Little Brown.

Martorella R., 1982. - The sociology of opera, New York, Praeger Publishers. 
Matheopoulos H., 1987. - Bravo : incontri con i grandi tenori, baritoni e bassi di oggi, Milano, Vallardi.

- 1992. - Diva : great sopranos and mezzos discuss their art, Boston, Northeastern University Press.

Mead G. H., 1963. - L'esprit, le soi et la société, Paris, Presses Universitaires de France.

Menger P.-M., 1989. - «Rationalité et incertitude de la vie d'artiste», L'Année sociologique, 39, pp. $111-151$.

- 1991. - «Marché du travail artistique et socialisation du risque : le cas des arts du spectacle », Revue française de sociologie, 32, 1, pp. 61-74.

- 1994. - «Appariement, risque et capital humain : l'emploi et la carrière dans les professions artistiques » dans P.-M. Menger, J. C. Passeron (éds.), L'art de la recherche : essais en l'honneur de Raymonde Moulin, Paris, La Documentation Française, pp. 219-238.

- 1997a. - La profession de comédien : formations, activités et carrières dans la démultiplication de soi, Paris, Ministère de la culture et de la communication.

- 1997b. - «L'activité du comédien : liens, indépendances et micro-organisations », Réseaux, 86, pp. 59-75.

Moindrot I., 1993a. - L'opéra seria ou le règne des castrats, Paris, Fayard.

- 1993b. - La représentation d'opéra : poétique et dramaturgie, Paris, Presses Universitaires de France.

Moulin R., 1967. - L'artiste, l'institution et le marché, Paris, Flammarion.

Pavis P., 1983. - «Production et réception au théâtre : la concrétisation du texte dramatique et spectaculaire », Revue des sciences humaines, 60, 189, pp. 51-88.

Pharo P., Quéré L. (éds.), 1990. - Les formes de l'action : sémantique et sociologie, Paris, Éditions de l'École des Hautes Études en Sciences Sociales.

Pleasants H., 1989. - Opera in crisis : tradition, present, future, London, Thames and Hudson.

Pullen R., Taylor S., 1996. - Montserrat Caballé : Casta Diva, Paris, Hachette.

Quéré L. (éd.), 1993. - La théorie de l'action : le sujet pratique en débat, Paris, Éditions du CNRS.

Quéré L., Relieu M., 1997. - «Les risques en situation d'incertitude», Paris, Centre d'étude des mouvements sociaux [texte non publié].

Rawls A., 1987. - «The interaction order sui generis : Goffman's contribution to social theory », Sociological theory, 5, pp. 136-149.

Relieu M., Quéré L., 1998. - «Mobilité, perception et sécurité dans les espaces publics urbains : comment se déplacer quand on ne voit plus les choses comme "tout le monde" ?", dans $\mathbf{H}$. Ansidei et al., Les risques urbains : acteurs, systèmes de prévention, Paris, Anthropos, pp. 91-120.

Richardson J. F., 1980. - «Career patterns of American opera singers» dans W. S. Herdon, A. J. MacDonald, J. L. Shanahan (eds.), Economic policy for the arts, Cambridge, Abt Books, pp. 176185.

Roselli J., 1984. - The opera industry in Italy from Cimarosa to Verdi : the role of the impresario, Cambridge, Cambridge University Press.

- 1997. - «From princely service to the open market : singers of Italian opera and their patrons, 1600-1850» dans R. Towse (ed.), Cultural economics: the arts, the heritage and the media industries, Chelterham, Edward Elgar, pp. 37-68.

Rosenthal H., Warrack J., 1995. - Guide de l'opéra, Paris, Fayard.

Saint-Pulgent M. de, 1991. - Le syndrome de l'opéra, Paris, Robert Laffont.

Salazar P.-J., 1980. - Idéologies de l'opéra, Paris, Presses Universitaires de France.

Santos F. P., 1976. - « Risk, uncertainty and the performing artist » dans M. Blaug (ed.), The economics of the arts, London, Martin Robertson, pp. 243-259.

Sarazin M., 1988. - Opéra de Paris : les mystères de la cathédrale de l'éphémère, Paris, Alain Moreau.

Strauss A. L., 1992. - Miroirs et masques : une introduction à l'interactionnisme, Paris, Métailié.

Sudnow D., 1993. - Ways of the hand : the organization of improvised conduct, Cambridge, MrT Press. 
Towse R., 1993. - Singers in the marketplace: the economics of the singing profession, Oxford, Clarendon Press.

- (ed.), 1997. - Cultural economics : the arts, the heritage and the media industries, Cheltenham, Edward Elgar.

Towse R., Khakee A. (eds.), 1992. - Cultural economics, Berlin, Springer-Verlag.

Turner R. H., 1962. - « Role-taking : process versus conformity » dans A. M. Rose, Human behavior and social processes : an interactionist approach, London, Routledge and Kegan Paul, pp. 20-40.

Urfalino P., 1990. - Quatre voix pour un Opéra : une histoire de l'Opéra-Bastille racontée par Michèle Audon, François Bloch-Lainé, Gérard Charlet, Michaël Dittman, Paris, Métailié.

Villers A., 1988. - «Réflexion sur les fondements théoriques d'une sociologie compréhensive de l'opéra », Revue de l'Institut de sociologie, 1-2, pp. 235-260.

Visnevskaja G., 1985. - Galina : histoire russe, Paris, Fayard.

Warren R., 1995. - Opera workshop : studies in understanding and interpretation, Aldershot, Scolar Press.

Willener A., 1997a. - La pyramide symphonique : exécuter, créer? Une sociologie des instrumentistes d'orchestre, Zürich, Seismo.

- 1997b. - Les instrumentistes d'orchestres symphoniques: variations diaboliques, Paris, L'Harmattan. 